\title{
Adenocarcinoma Mucinoso de Uraco y Pseudomixoma Peritoneal
}

\author{
M. Soto Delgado, *G. Pedrero Márquez, C. Varo Solís, F.O. Rodríguez-Rubio Cortadellas, \\ C. Sánchez Bernal, D. González Moreno
}

Servicio De Urología. * Unidad de Enfermería. Hospital Universitario de Puerto Real. Cádiz.

Actas Urol Esp 2006; 30 (2): 222-226

\section{RESUMEN \\ ADENOCARCINOMA MUCINOSO DE URACO Y PSEUDOMIXOMA PERITONEAL. MUCINOUS ADENOCARCINOMA OF THE URACHUS AND PERITONEAL PSEUDOMYXOMA.}

$\mathrm{El}$ adenocarcinoma de uraco es un tumor extremadamente raro, con una incidencia de 1/5.000.000 de habitantes, lo que representa menos del 0,001 de todos los tumores de vejiga.

El pseudomixoma peritoneal es una neoplasia rara caracterizada por ascitis mucinosa que envuelve la superficie peritoneal y el omento. Usualmente está asociada con tumores mucinosos benignos o malignos del apéndice $u$ ovario.

En este trabajo, presentamos un caso de pseudomixoma peritoneal causado por un adenocarcinoma mucinoso de uraco.

Palabras Clave. Adenocarcinoma Mucinoso. Uraco. Pseudomixoma Peritoneal.

\section{ABSTRACT}

AB MUCINOUS ADENOCARCINOMA OF THE URACHUS AND PERITONEAL PSEUDOMYXOMA

The adenocarcinoma of the urachus is very rare tumor, with an incidence of 1/5.000.000 inhabitants, represents less than 0,001 of all types of bladder cancer.

Peritoneal pseudomixoma is a rare neoplasm characterized by mucinous acites that involvement the peritoneal surface and omentum. Usually is associated with benign o malignant mucinous tumor of the appendix or ovary.

In this paper, we present a case of peritoneal pseudomixoma caused by a mucinous adenocarcinoma of the urachus.

Keywords. Mucinous Adenocarcinoma. Urachus. Peritoneal Pseudomixoma.

$\mathrm{L}$

os tumores de uraco son tumores muy raros, representando menos del $0,5 \%$ de los tumores de la vejiga, siendo más frecuentes en varones y entre la $5^{\underline{a}-7^{\mathrm{a}}}$ décadas de la vida. Alrededor de un $90 \%$ de ellos son adenocarcinomas mucinosos bien diferenciados aunque muy infiltrantes, cursando con síntomas poco específicos. Esto hace que en la mayor parte de los casos el tumor se encuentre en un estadio avanzado en el momento del diagnóstico, por lo que la supervivencia en general es corta ${ }^{1}$.

El pseudomixoma peritoneal es una entidad clínica rara que se caracteriza por la ocupación de la cavidad peritoneal por grandes colecciones de contenido mucinoso y ascitis que envuelve la superficie peritoneal ${ }^{2}$. Actualmente se describe mayoritariamente en asociación con tumores malignos bien diferenciados o de malignidad en el límite. En la mayoría de los casos el tumor primario se localiza a nivel del ovario o apéndice, aunque también se ha descrito en relación con tumores de otras localizaciones ${ }^{3}$.

En este trabajo presentamos un caso clínico donde se asocian dos patologías extremadamente raras: adenocarcinoma mucinoso de uraco y pseudomixoma peritoneal. 


\section{CASO CLÍNICO}

$\mathrm{El}$ paciente era un varón de 56 años de edad, sin antecedentes personales de interés, que consulta por episodios autolimitados de hematuria macroscópica monosintomática de 2 meses de evolución.

Al tacto rectal, la próstata estaba bien delimitada y era de consistencia adenomatosa (grado II/IV).

Las exploraciones complementarias realizadas fueron las siguientes:

- Hemograma, bioquímica, urocultivo, citología de orina y sedimento: dentro de la normalidad. PSA: $1,46 \mathrm{ng} / \mathrm{ml}$.

- Ecografía urológica: quistes sinusales renales bilaterales. Vejiga normal.

- Urografia intravenosa (Fig. 1): distorsión pielocalicial por los quistes antes mencionados. Uréteres permeables. Vejiga normal. En la placa posmiccional llamaba la atención la presencia de una "fuga de contraste" hacia la cavidad abdominal (Fig. 2).

- Cistoscopia: masa de aspecto sólido de pequeño tamaño localizada en cúpula vesical.

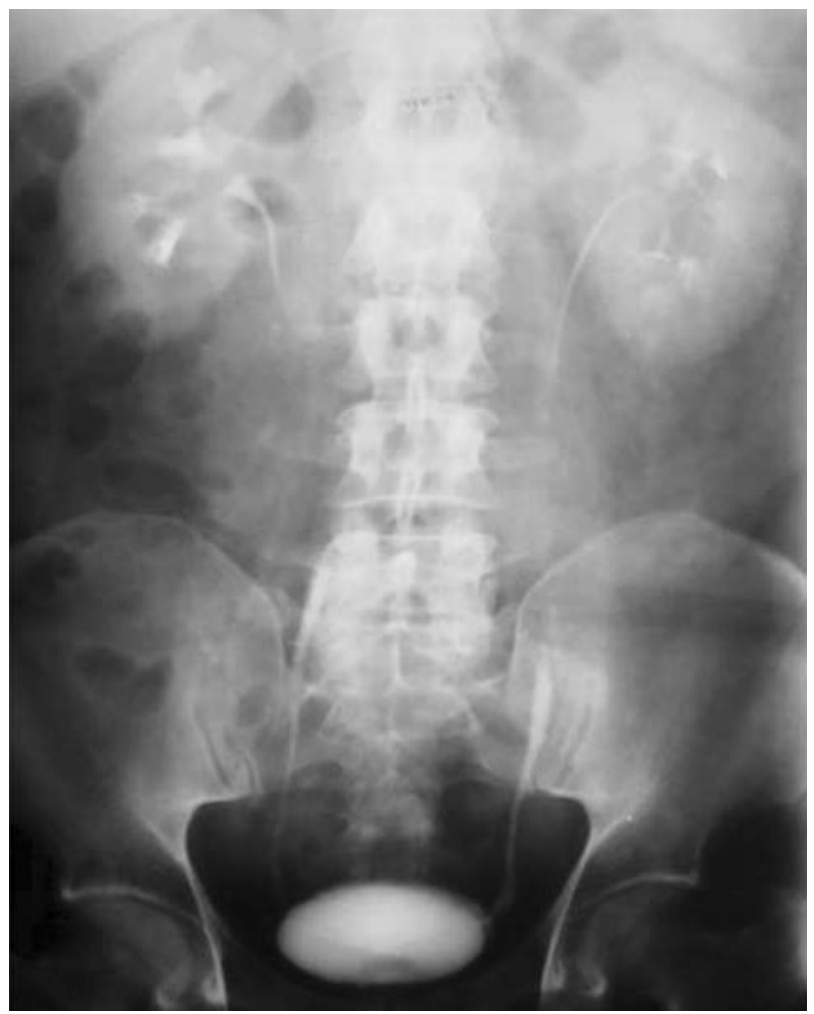

FIGURA 1. Urografia intravenosa.

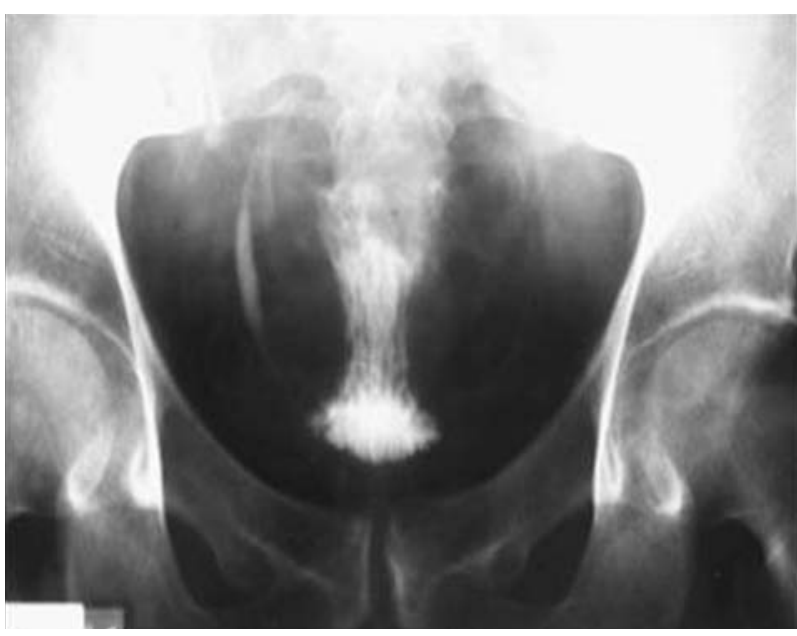

FIGURA 2: Placa posmiccional

Ante estos hallazgos se procedió a la resección transuretral (RTU) de la lesión, con el resultado histopatológico de adenocarcinoma que infiltra la pared vesical.

Tras este diagnóstico se realiza estudio de extensión mediante radiografía de tórax (que es informada como normal) y TAC abdominopélvico donde se objetiva una estructura tubular que se extiende desde la cúpula vesical hasta el ombligo con pared gruesa sobre todo cercana a vejiga (uraco persistente con posible infiltración de la pared por tumor) además de moderada cantidad de líquido libre en cavidad peritoneal (Fig. 3).

Una semana antes del tratamiento quirúrgico definitivo, el paciente ingresó por cuadro de fiebre alta acompañada de síntomas miccionales, dolor abdominal, vómitos y anorexia.

A la exploración física, el paciente presentaba distensión abdominal con cierto grado de irrita-

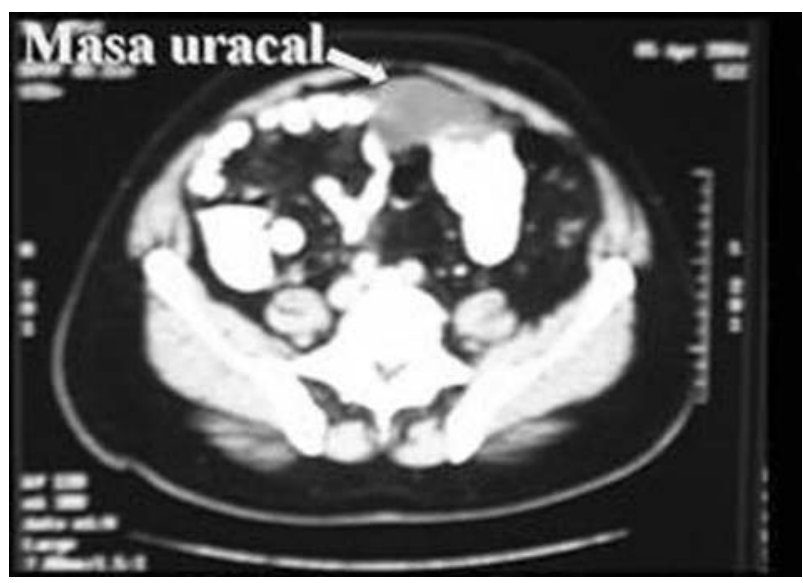

FIGURA 3: TAC. 
ción peritoneal. En la analítica destacaba la presencia de una leucocitosis con neutrofilia. Tras instaurar tratamiento antibiótico parenteral de amplio espectro, el paciente mejoró de su sintomatología a excepción de la distensión abdominal. En la ecografia abdominal realizada se objetivaba una gran cantidad de líquido ascítico con tabiques en su interior.

Con la sospecha diagnostica de adenocarcinoma de uraco se realizó una cistectomía parcial amplia que incluía la cúpula vesical, gran masa a nivel de uraco y ombligo, además de una linfadenectomía pélvica. Durante el acto quirúrgico objetivamos una gran cantidad de material mucoide infiltrando toda la cavidad peritoneal (se tomaron varias muestras de distintas localizaciones para estudio histológico). El diagnóstico anatomopatológico de la pieza quirúrgica fue de adenocarcinoma mucinoso de uraco con extensión a vejiga urinaria y peritoneo (Fig. 4). El informe histológico del material mucoide enviado fue de pseudomixoma peritoneal (Fig. 5).

En la actualidad, después de 1 año de seguimiento, el paciente se encuentra asintomático y con estudio de extensión libre de enfermedad.

\section{DISCUSIÓN}

La mayoria de las patologias uracales son debidas a la persistencia de este conducto que en la etapa embrionaria une la cúpula vesical con la alantoides y que al obliterarse tras el nacimiento da lugar al ligamento umbilical medio. Este con-

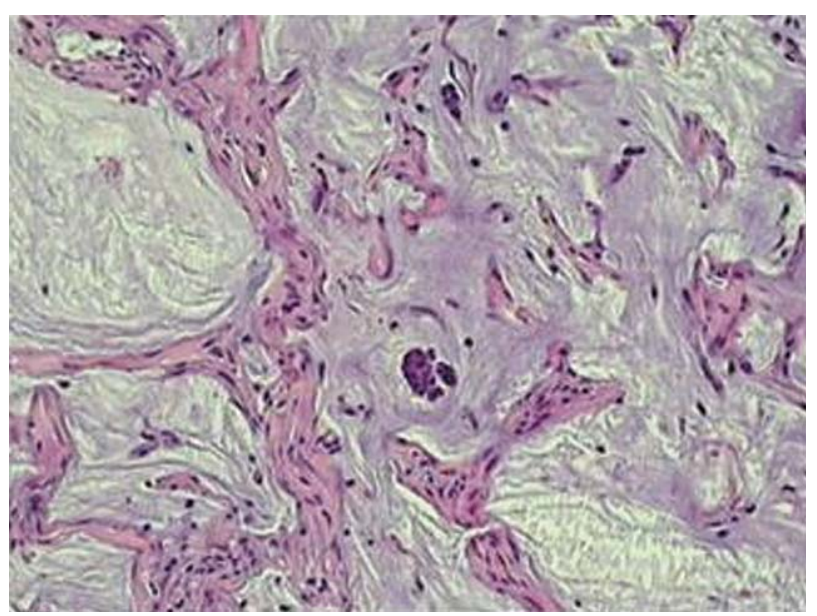

FIGURA 4: A nivel peritoneal se observa material mucoide que diseca los haces de colágeno, en el que sobrenadan grupos papiliformes (pseudomixoma peritoneal).

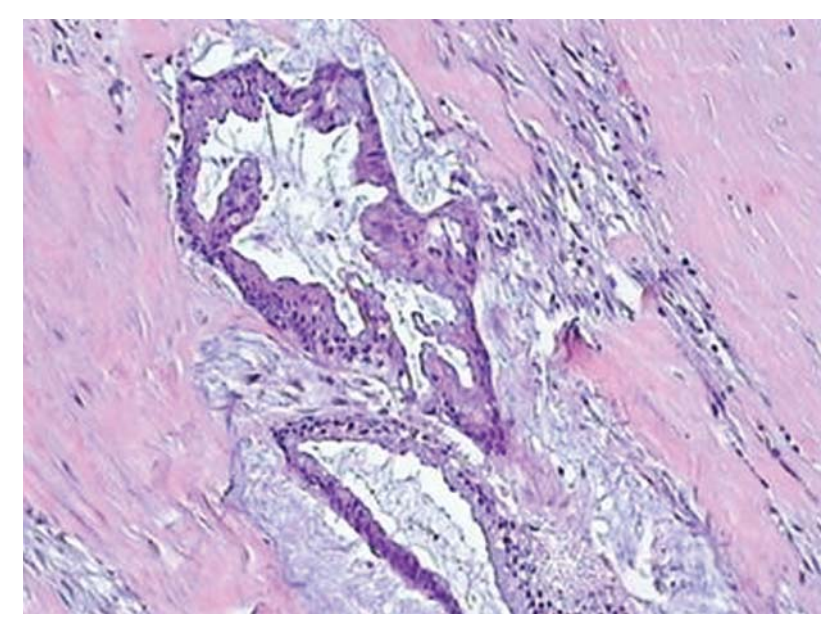

FIGURA 5: Se observa una proliferación neoplásica glandular de aspecto enterico, mucinosa.

ducto tiene tres porciones, intramucosa, intramuscular y supravesical, y el epitelio que reviste su luz es de tipo transicional, aunque a veces sufre cambios de metaplasia glandular. En series amplias de autopsia se reconocen en un 30-70\% de los casos restos de uraco, siendo ésta la causa de alteraciones como quistes, fístulas e infecciones. Los tumores son muy raros, pudiendo ser benignos (principalmente adenomas) o malignos. Dentro de estos últimos, los adenocarcinomas de tipo mucinoso suponen alrededor de un $70 \%$ del total ${ }^{1}$.

El carcinoma de uraco tiene tendencia a la invasión local y no suele dar síntomas clínicos hasta que el tumor invade las estructuras vecinas ${ }^{4}$. El síntoma más frecuente es la hematuria, debido a la erosión de la cúpula vesical ${ }^{5,6}$. Otros síntomas como la disuria, dolor en hipogastrio o moco en la orina, son menos frecuentes $^{6,7}$. Este tipo de tumores se extienden por el espacio de Retzius, la pared abdominal anterior y el peritoneo ${ }^{4}$.

La actitud inicial ante un posible tumor de uraco es idéntica a la que se manifiesta para diagnosticar el resto de los tumores de vejiga. La cistoscopia y la resección transuretral son dos técnicas esenciales, tanto para determinar la situación de tumor en la vejiga como para hacer el diagnóstico histopatológico. Desde el punto de vista de las técnicas de imagen, las radiografías simples suelen ser normales y la urografía intravenosa rara vez presenta un defecto de replección en la cúpula vesical en 
estadios precoces. La Tomografía Axial Computarizada (TAC) y la Resonancia Magnética Nuclear (RMN) son las pruebas radiológicas más específicas para hacer el diagnóstico y hacer el estadiaje de este tipo de tumores ${ }^{4}$.

Sheldon en 1984 propuso el siguiente sistema para clasificar y estadiar a los adenocarcinomas de uraco $^{8}$ :

Estadio I: Tumor localizado en la mucosa uracal.

Estadio II: Tumor que invade la submucosa o la capa muscular del uraco, pero que se encuentra confinado en el uraco.

Estadio III: Tumor que se extiende fuera del uraco. III-A: a la vejiga; III-B: a la pared abdominal; III-C: al peritoneo; III-D: a alguna víscera diferente.

Estadio IV: Tumor con metástasis. IV-A: afectación de los ganglios linfáticos regionales; IV-B: metástasis a distancia.

La cirugía constituye la mejor opción de tratamiento. La mayoría de los autores son partidarios de una cistectomía parcial extensa que incluya el ombligo, el uraco con el peritoneo, la cúpula de la vejiga y los ganglios pélvicos (linfadenectomía pélvica bilateral) ${ }^{7-9}$.

El pronóstico no es bueno ya que la mayoría de estos tumores se diagnostican en estadio III (en nuestro caso, el estadio de la enfermedad era III-C). Se estima que la supervivencia global a los 5 años con cualquier tipo de tratamiento quirúrgico asignado, cistectomía radical o cistectomía parcial amplia, está entre el 43 y el 50\%. La radioterapia (la mayoría de estos tumores son radio-resistentes) y la quimioterapia adyuvante a la cirugía no han conseguido mejorar la supervivencia ${ }^{4}$.

El pseudomixoma peritoneal es un proceso muy raro, y a pesar de que el caso que nosotros presentamos es el de un varón, tiene preponderancia en mujeres con una media de presentación de aproximadamente 55 años. En la mayoría de los casos se describe la existencia de un tumor primario, generalmente un cistoadenoma o cistoadenocarcinoma a nivel de ovario (45\%) o de apéndice cecal (29\%). Se han descrito casos además en relación con tumores colon, endometrio, páncreas, vía biliar, mama y tumores de uraco, no siendo posible identificar el tumor originario sólo en un pequeño porcentaje de ca$\operatorname{sos}^{3,10}$.

La presentación clínica más frecuente de este proceso es el dolor y la distensión abdominal. Otros síntomas acompañantes son anorexia, naúseas, vómitos, pérdida de peso, astenia, síntomas urinarios y diarrea ${ }^{3,10}$ ).

La exploración física muestra generalmente la presencia de distensión abdominal, siendo posible encontrar también fiebre e irritación peritoneal. En la analítica se puede presentar leucocitosis y/o anemia ${ }^{2}$.

Además de por la sospecha clínica, el diagnóstico preoperatorio se realiza fundamentalmente por técnicas de imagen como la ecografia y TAC abdominales. La ecografía puede mostrar como hallazgos típicos la existencia de una manto ecogénico con colecciones intraperitoneales anecoicas de naturaleza quística, desplazamiento de asas intestinales y presencia de ascitis. En la TAC se aprecia ascitis, quistes peritoneales con tabiques, calcificaciones y compresión extrínseca de las vísceras ${ }^{11}$.

El diagnóstico definitivo se obtiene durante la intervención quirúrgica y con el estudio anatomopatológico ${ }^{12}$.

La invasión local es una constante. Sin embargo, la metastatización a distancia ha sido descrita sólo en casos aislados, a nivel de vértebras, cavidad torácica y nódulos linfáticos ${ }^{3}$.

El tratamiento fundamental del pseudomixoma peritoneal es la resección tumoral extensa junto con la administración de quimioterapia intraperitoneal perioperatoria, intraoperatoria y postoperatoria precoz para conseguir la erradicación de la enfermedad microscópica ${ }^{10}$.

La recurrencia del cuadro es frecuente $(76 \%$ de los casos), estando indicada nuevamente la resección quirúrgica como la mejor terapéutica. La supervivencia a los 5 años se estima en un $54 \%$ y a los 10 años en el 18-34\% según los autores $^{3}$. En nuestro caso, y tras un año de seguimiento, el paciente se encuentra libre de enfermedad. 


\section{REFERENCIAS}

1. Álvarez C, Sánchez JM, Busto L, Pombo F, Arnal F. Adenocarcinoma mucinoso de uraco sincrónico con adenocarcinoma colorrectal. Valor de la inmunohistoquímica en el diagnóstico diferencial. Actas Urol Esp 1998;22:515-518.

2. Hinson FL, Ambrose NS. Pseudomyxoma peritonei. Br J Surg 1998;85(10): 1332-1339.

3. Gough DB, Donohue JH, Schutt AJ, Gonchorrott N, Goellner JR, Wilson TO, et al. Pseudomyxoma peritonei: long-term patient survival with an aggresive al approach. Ann Surg 1994; 219:112-119.

4. Ojea A, Núñez A, Domínguez F, Alonso A, Rodríguez B, Benavente $\mathrm{J}$, et al. Adenocarcinoma mucinoso de uraco. Actas Urol Esp 2003;27(2):142-146.

5. Pantuck AJ, Bancila E, Das KM, Amenta PS, Cummings KB, Marks M, Weiss RE. Adenocarcinoma of the urachus and bladder expresses unique epithelium epitope: an immunohistochemical study. J Urol 1997;158(5):1722-1727.

6. Henly DR, Farow GM, Zinke H. Urachal cancer. Rol of conmservative surgery. Urology 1993;42(6):635-639.

7. Herr HW. Urachal carcinoma: the case for extendede partial cystectomy. J Urol 1994; 151(2):365-366.
8. Sheldon CA, Clayman RV, Gonzalez R, Williams RD, Fraley EE. Malignant urachal lesions. J Urol 1984;131(1):1-8.

9. Santucci RA, True LD, Lange PH. Is partial cystectomy the treatment of choice for mucinous adenocarcinoma of the urachus?. Urology 1997;49(4):536-540.

10. Wertheim I, Fleischhacker D, Mclachlin CM, Rice LW, Berkowitz RS, Gott BA. Pseudomyxoma peritonei: a review of 23 cases. Obstet Gynecol 1994;84(1):17-21.

11. Guillarte J, Cantero J, Mantas JA. Hallazgos ecográficos y tomográficos en el pseudomixoma peritoneal. Rev Esp Enf Digest 1997;89:798-799.

12. Mulvany N, Ooi K: Pseudomyxoma peritonei: a cytohistopathologic study of nine cases. Diagn Cytopathol 1996; 15(2): 144-150.

Dr. M. Soto Delgado.

(Trabajo recibido el 6 de mayo de 2005) 